https://doi.org/10.48009/2_iis_2005_352-357

\title{
THE CO-EXISTENCE OF IPV6 AND IPV4 IN THE U.S.A
}

\author{
Dr. Garry L. White, Texas State University - San Marcos, gw06@business.txstate.edu
}

\begin{abstract}
There is speculation that by the year 2010 all IPv4 Internet addresses will be used up. By then, Asia and Europe plan to be using the new IPv6 Internet addresses. The computer industry literature indicates that the U.S.A. is lagging behind with the implementation of IPv6. Although there is not a shortage with IPv4 addresses in the U.S.A., new technologies will cause such a shortage. Such technologies are mobile phones, wireless communications, voice/video transmissions, and connecting household appliances to the Internet. While IPv4 was design to handle text data, IPv6 is better suited to handle voice/video data and mobile nodes. These new technical demands will require the implementation of IPv6 in the U.S.A. However, there will still be a tendency to use IPv4 in the U.S.A., especially for web pages and e-mail. Through the use of tunneling and translation of IP addresses, IPv6 will co-exist with IPv4. Total conversion to IPv6 in the U.S.A. will be several years after 2010.
\end{abstract}

Keywords: IPv6, IPv4, NAT, U.S.A., Internet Protocol

\section{INTRODUCTION - THE NEED}

The Internet of today is no longer just servers and PCs. There are other mobile devices such as personal digital assistants, cell phones, and automobiles $[2,13,20]$. In the past, data packets could be delayed and arrive out of sequence in transmission over hard wire. Examples are e-mail and web pages. Today real-time voice/video wireless mobile devices are appearing on the Internet. These new devices are unable to tolerate delays and out of sequence transmissions as found with e-mails and web pages. IPv6 can deal with these new devices on the Internet.

These new devices have accelerated the need for more IP addresses [6, 12]. Information Technology vendors argue that IP addresses will disappear as the Internet enables these devices [6]. The problem is that the IPv4 Internet was never designed for these new devices. IPv4 and it's address scheme were designed for digital transmissions of ASCII text code between stationary and wired connected PCs. These IPv4 addresses will be depleted by 2010 [37]. That is when many speculate the Internet will be close to $100 \%$ compliant with the new Internet protocol, IPv6 [16, 39].

\section{REASONS WHY IPV4 IS INHIBITING IPV6 IN THE U.S.A.}

The movement to IPv6 in Europe and Asia is due to a shortage of IPv4 addresses [14, 22, 26]. However this is not the case for the U.S.A. In 2002, 70\% to 74\% of all IPv4 addresses have been allocated to organizations located in North America [12, 44]. For example, Stanford University and MIT each have more IP addresses then the entire People's Republic of China. [33].

Also, the problem of IPv4 address shortages has been less then first indicated due to other technologies. Protocols such as Network Address Translation (NAT) and Dynamic Host 
Configuration Protocol (DHCP) have been able to increase the number of nodes on the Internet with a limited number of IPv4 addresses [40]. For example, NAT technologies link many networked devices to the Internet through a translation device with a single IPv4 address [6]. This single IPv4 address, along with port numbers, can be translated into internal private IP addresses. RFC 1918 defines within the IPv4 address scheme private addresses that can be duplicated in different networks [27]. These addresses are never assigned to any organization as a registered network number. NAT also uses port numbers in translation from external node addresses to internal node addresses. An outside computer can crate three connections via one IPv4 address with three different port numbers. When these connections go through a NAT device, they are translated to three different internal private addresses. Fortune 500 companies are accustomed to having 30 or 40 public IP addresses and using NAT for everything else [35]. NAT breaks the limit of 3.7 billion IPv4 addresses [41].

DHCP assigns temporary IPv4 addresses to nodes when the nodes login. If a node is not in use, there is no need to assign an IP address to it. Therefore, IP addresses used are based on the number of connections instead of the number of physical computers.

These technologies of NAT and DHCP have lessened the need to migrate to IPv6, especially for the U.S.A. IPv4 provides corporations with all the network functionality they need at a lower cost [45]. There are those who believe the world is adequately served by IPv4 [41].

\section{PROBLEMS WITH IPV6 MIGRATION}

Problems to migrate to IPv6 involve a large amount of learning, training, and restructuring [8, 11]. Applications will need to be rewritten to deal with the increased size of an IPv6 address. DNS servers and complex applications such as databases, firewalls, browsers, and IP phones will have to be upgraded [8, 19, 36]. Filtering and Intrusion Detection System (IDS) security for IPv6 are not as developed as IPv4 [11]. The cost of changing to IPv6 is tremendous [35]. And, as some believe, there is no current need $[17,22]$.

Since there are plenty of IP addresses in the U.S.A. and a big investment in IPv4 infrastructure, many American companies fail to see IPv6 as a priority $[12,22]$. Therefore, U.S.A. companies lack interest for IPv6 [14, 30, 34].

\section{THE IPV6 ADVANTAGES}

There are problems with IPv4's, especially with the use of NAT to increase the number of node addresses. NAT maybe unable to meet the needs of very large networks that serve 50 to 100 million IP-enabled devices. Address translation also may result in higher network capital and operational costs [37]. Another problem with NAT is a potential single point of failure into the network. NAT also disables many existing network applications that require unique addresses, such as H.323 conferencing [31]. Although NAT works well with web and e-mail; it does NOT work well with voice over IP, IPsec, and real-time video [21]. Translation of addresses causes a delay for time sensitive data such as audio and video. Other problems with IPv4 are lacking global address space [31] and lacking the ability to provide quality service for real-time packets [44]. 
IPv6 resolves many of these IPv4 problems: lack of addresses and lack of hierarchy levels in IP address structure for better performance-management, security, and automatic address assignment $[4,25]$. IPv6 has four advantages over IPv4:

1) IPv6 dramatically increases the IP address size. It also supports more levels of addressing hierarchy making routing more efficient [15].

2) Extension headers for authentication, privacy capabilities, security, and integrity are included [10].

3) Quality of Service capabilities provides easier management of real-time file-sharing and voice services [44]. Multimedia applications will become common on the Internet.

4) Automatic configuration of IPv6 allows a host on the Internet to generate a unique IP address for itself based on network topology. It allows a customer/user to change service providers without having to manually reconfigure addresses for every node on the customer/user network. It also makes using roaming mobile hosts, such as Internet-enabled cellular telephone, easier [23, 40].

These characteristics make IPv6 wireless/mobile/audio/visual friendly. Where as, IPv4 is unfriendly to these new Internet features. IPv4 was designed for wired digital computers using ASCII text data.

\section{THE NEED FOR IPV6: WIRELESS/MOBILE DEVICES}

Problems with IPv6 deployment include the tremendous cost of changing to IPv6 [35]. There is a lack of empirical evidence showing IPv6 is better than IPv4 [22]. Many network managers have decided to wait and see what happens with IPv6 [6].

To drive a transition to IPv6 a key application is needed [41]. That key application is wireless networks/data communications [9]. Wireless communication vendors support IPv6 [24]. They have committed to IPv6 for their third generation standards. [14, 24, 29]. And mobile data networks are the principal beneficiaries and motivators of IPv6 [38]. For example, cell phones are becoming Internet-enabled $[1,5]$. Providers of next-generation mobile digital telephony services point out they'll need IP addresses for millions of devices for data networking [21]. It is predicted the world will totally integrated with wireless technology sometime between 2012 and 2022 [18]. These mobile data network vendors are the principal beneficiaries and therefore are the motivators of IPv6 [38]. IPv6 deals with wireless connectivity and will resolve the address problem due to so many devices on the Internet [9]. Already there are several U.S.A. sites providing IPv6 connection $[3,11]$. Development of IPv6 wireless in the U.S.A. is speculated to begin in 2006 [11].

\section{CO-EXISTENCE OF IPV4 \& IPV6}

Equipment and operating system vendors have begun supporting IPv6 along with manufacturers of devices that use wireless devices [20]. IPv6 is currently available in hardware and software. Sun, 3Com, Cisco routers, Juniper routers, and Nortel already support IPv6 [8, 19]. IPv6 stacks are available for operating systems from Apple Computer, Linux, Microsoft, and Sun 
Microsystems [8]. Over 185 U.S.A. sites have registered with 6bone [11]. Several IPv6 exchanges also now exist in the U.S.A. [3].

The technology exists where IPv4 and IPv6 can co-exist. These technologies include [4]:

1) Dual stack mechanisms- is based on the usage of a DHCPv6 server, which temporarily assigns global IPv4 addresses to IPv6 hosts that communicate with IPv4 hosts.

2) Tunneling mechanisms- IPv6 packets are encapsulated into an IPv4 packet and is transmitted over an IPv4 network.

3) Translation mechanism- IPv6/IPv4 networks translate IPv6 addresses/headers to IPv4 addresses/headers and vice-versa.

Already the PAIX exchange in Palo Alto, California, has been providing both IPv4 and IPv6 since October, 2000 [3].

\section{SUMMARY}

With the increase demand for IP-addressable devices (cell phones, game players, etc.); the prospects for IPv6 are improving [28, 44]. Equipment and operating system vendors have begun supporting IPv6 along with manufacturers of devices that use "wireless" and embedded devices [20]. However, many U. S. based vendors are not heavily supporting IPv6. Interest and market demand in the U.S.A. are relatively low [11]. Many do not want to spend the time and money necessary to migrate to IPv6 until they need to [20].

Will U.S. providers have enough addresses to implement their wireless Internet capabilities? IPv4 development did not take into account wireless' needs. And as IP addresses begin to be used by devices such as appliances with Internet connections, the current availability of IPv4 will be inadequate [43]. Address space will be depleted between 2006 and 2011 [25]. To address the shortage of IP addresses due to new wireless/mobile devices, IPv6 will be implemented for these new devices. This will avoid the burden of conversion of current servers and PCs from IPv4 to IPv6.

\section{CONCLUSION}

Today the Internet uses IPv4 rules of communication and node addresses. However, IPv4 addresses will be used up sometime before 2005 and 2011 [12, 32, 37, 40, 44]. That is when many speculate the Internet will be close to 100\% compliant with the new Internet protocol, IPv6 $[7,16,39]$. This may not be the case of the U.S.A.[42].

Literature from the computer industry suggests that when the world is using IPv6 in 2010, the U.S.A. will be lagging behind. Because there is not a critical need for total conversion to IPv6 in the USA, it appears that the USA will continue to use IPv4 for most non-wireless Internet applications way beyond 2010. The author speculates that IPv6 will be used in the USA for realtime mobile voice/video devices in the more immediate future. The U.S.A. will also use IPv4 for web pages, e-mail, web servers and stationary PCs. There will be a co-existence with IPv6 and 
IPv4 in the U.S.A. for a much longer time after Europe and Asia are totally IPv6. Unfortunately, as Latif Ladid, President of the IPv6 Forum, said at the 2001 Global IPv6 Summit in Soul, Korea, said "the longer the upgrade is postponed, the costlier it will be and more complex transition compared to Y2K" [18].

\section{REFERENCES}

1. Barney, D. \& Marsan, C. D. (1999). Internet pioneer: IPv6 transition needed. Network World, 16(43), 16.

2. Blagi, S. \& Ryan, V. (2000). The new and improved IP. Telephony, 238(19), 36-46.

3. Blanchet, M. (2003). IPv6 Exchanges Web Site. www.v6nap.net. Accessed 12/5/2003.

4. Bouras, C., Ganos, P. \& Karaliotas, A. (2003). The deployment of IPv6 in an IPv4 world and transition strategies. Internet Research: Electronic Networking Applications and Policy, 13(2), 86-93.

5. Chao, H.,Chu, Y. \& Lin, M. (2000). The implication of the next-generation wireless network design: Cellular mobile IPv6. IEEE Transactions on Consumer Electronics, 46(3), 656-63.

6. Cope, J. (May, 2001). IPv6: Is it inevitable? Computerworld, 35(22), 58-59.

7. Deering, S. (April, 2002). IPv6 Overview \& Status Report. Presentation to the Amsterdam Science and Technology Center. http://isoc.nl/activ/cursusmateriaal/2002-Masterclass-IETFIPv6.ppt. Accessed 9/5/02.

8. DeMaria, M. J. (Feb, 2002). Slow road to IPv6. Network Computing, 13(3), 83-85.

9. Fontana, J., Marsan, C. D., Greene, T. \& Cox, J. et al. (2000). Power Prognosticator. Network World, 17(1), 27-32.

10. Goncalves, M. \& Niles, K. (1998). IPv6 Networks. New York: McGrew-Hill.

11. Grossetete, P. (2002). IPv6 in the U.S.A. Presentation at the Global IPv6 Summit in Japan 2002, Pacifico Yokohma, Japan. www.soi.wide.ad.jp/class/20020035/slides/06/1.html. Accessed 12/5/2003.

12. Gwin, P. (Jun, 2002). Upgrading the Internet and the IPv6 debate. Europe, (417), 5.

13. Harpster, S. (2000). Ready or not: IPv6 is coming. Embedded Systems Programming, 13, 9598.

14. Higgins, K. J. (Nov, 2000). IP's Next Stop. InfoWorld, 59-62.

15. Hinden, R. M. (June, 1996). IP Next Generation Overview. Communications of the ACM, $39(6), 62-71$.

16. Hwany, C.J. (July, 2001). IPv6 Policy Implications in Korea. Global IPv6 Summit, Soul, Korea. http://www.ipv6.or.kr/ip6summit. Accessed 9/5/02.

17. Koprowski, G. (1998). Emerging uncertainty over IPv6. Computer, 31(11), 16-17.

18. Ladid, L. (July, 2001). IPv6 Deployment Strategies in Europe. Global IPv6 Summit, Soul, Korea. http://www.ipv6.or.kr/ip6summit. Accessed 9/5/02.

19. Lawson, S., Miyake, K. \& Evers, J. (Feb, 2002). IPv6 enters the real world. InfoWorld, 24(7), 35-36.

20. Lawton, G. (Aug, 2001). Is IPv6 finally gaining ground? Computer, 34(8), 11-15.

21. Loshin, P. (Oct, 1999). IPv6 over everything. Data Communications, 28(15), 41-46.

22. Loshin, P. (Jun, 2000). IPv6. Boardwatch Magazine, 14(6), 94-98.

23. Marson, C. D. (Aug, 1999). Why the push for support of IPv6? Network World, 16(35), 16.

24. Marsan, C. D. (Oct, 2000). Wireless boosting IPv6. Network World, 17(43), 1-2. 
25. Marsan, C. D. (Jul, 2001). ISP group at vortex of IPv6 transition. Network World, 18(30), 2526.

26. Morrison, G. (Apr, 2002). The IPv6 Factor. Electronic News, 48(15), 24.

27. Odom, W. (2004). CCNA Self-Study: CCNA ICND Exam Certification Guide. Cisco Press, Indianapolis, IN. p. 259.

28. Passmore, D. (May, 2002). Next-gen router prospects. Business communications Review, 32(5), 16-17.

29. Paulson, L. D. (Jan, 2001). Will wireless be IPv6's killer app? Computer, 34(1), 28-29.

30. Petrosky, M. (2001). IPv6: this is your wake-up call. Network World, 18(20), 41-42.

31. Quinn, B. (Mar, 2000). IPv6 is inevitable. Midrange Systems, 13(4), 48.

32. Salus, P. (Jan, 2000). Penguin's Progress: A Look at IPv6. Linux Journal, 2000(69es)

33. Schwartz, E. (Jul, 2002). IPv6 stands to level playing field. InfoWorld, 24(30), 23.

34. Snyder, J. (Sept, 1999). Stillborn IPv6 offers no compelling reasons to switch. Network World, 16(39), 37.

35. Snyder, J. (Mar, 2000). Why IPv6 will never come. Midrange Systems, 13(4), 47.

36. Steinke, S. (Jul, 2001). Whither IPv6? Network Magazine, 16(7), 10.

37. Suomela, P. (Dec, 2001). Growing into IPv6. Wireless Review, 18(23), 10.

38. Sweeney, D. (Apr, 2002). Comin' on slow. America's Network, 106(5), 20.

39. Talley, B. (Aug, 1998). IPv6 cuts address chaos. InfoWorld, August 24, 1998, p.61-62

40. Watlton, C. (May, 1999). IPv6: At the Starting Line. NetWare Connection, 6-17.

41. Weiser, M. (Sept, 2001). Whatever happened to the Next-Generation Internet? Communications of the ACM, 44(9), 61-68.

42. White, G. (March, 2003). Internet Technology in 2010: An Issue for the U.S.A. Proceedings of Southwest Decision Sciences Institute $34^{\text {rd }}$ Annual Conference, Houston, TX.

43. Wickham, R. L. (Jun, 2000). IPv6 adds value to IP address issue. Wireless Review, 17(11), 14.

44. Wieland, K. (May, 2002). Addressing the IPv6 Issue. Telecommunications International, 36(5), 27-30.

45. Yoke, C. (May, 2002). Two years later, still sticking with IPv4. Network World, 19(19), 47. 